EPJ Web of Conferences 116, 06003 (2016)

DOI: $10.1051 /$ epjconf/201611606003

(C) Owned by the authors, published by EDP Sciences, 2016

\title{
Calibration methods and tools for KM3NeT
}

\author{
Vladimir Kulikovskiy ${ }^{\mathrm{a}}$ on behalf of the KM3NeT collaboration \\ INFN, Laboratori Nazionali del Sud (LNS), via S. Sofia 62, 95123 Catania, Italy
}

\begin{abstract}
The KM3NeT detectors, ARCA and ORCA, composed of several thousands digital optical modules, are in the process of their realization in the Mediterranean Sea. Each optical module contains 313 -inch photomultipliers. Readout of the optical modules and other detector components is synchronized at the level of sub-nanoseconds. The position of the module is measured by acoustic piezo detectors inside the module and external acoustic emitters installed on the bottom of the sea. The orientation of the module is obtained with an internal attitude and heading reference system chip. Detector calibration, i.e. timing, positioning and sea-water properties, is overviewed in this talk and discussed in detail in this conference. Results of the procedure applied to the first detector unit ready for installation in the deep sea will be shown.
\end{abstract}

\section{Introduction}

The KM3NeT detectors [1] are large 3-dimensional arrays of optical modules. Digital optical modules (DOMs) are arranged by modulo eighteen on the flexible lines attached to the sea bottom. Each line has a base module close to the anchor which performs the line power control and the optical link signal amplification. For the neutrino events reconstruction with tracking precision better than $1^{\circ}$, the optical modules need to be synchronized with sub-nanosecond precision and their position determined with better than meter precision. Each DOM and the base have an identical Control Logic Boards which perform the signals processing and transfer, time synchronization and control of the instruments.

The detector is connected to the shore via the main electro-optical cable. The single fiber in the cable (about $40 \mathrm{~km}$ in Toulon, and $100 \mathrm{~km}$ in Capo Passero) is common for the base and the DOMs. For the base synchronisation a fully compliant White Rabbit ${ }^{1}$ system was chosen. White Rabbit is developed to synchronise the clocks between the nodes with a bidirectional point to point optical fiber connection. The system continuously monitors the delay of the fiber connecting the nodes and keeps the node clocks synchronus. The current KM3NeT optical network architecture does not allow White Rabbit synchronisation for the DOMs. A custom protocol was developed for their synchronisation. Time and slow control broadcast from the shore master White Rabbit switch is done to all DOMs, while data return is performed to normal data switch. Synchronisation is achieved thanks to the fixed electronic latencies in the system. The most important delay in the system (main electro-optical cable) is measured continiously by the shore - line base synchronisation system [3]. Time synchronisation

\footnotetext{
a e-mail: kulikovs@ge.infn.it

${ }^{1}$ http://www.ohwr.org/projects/white-rabbit/wiki/
}

This is an Open Access article distributed under the terms of the Creative Commons Attribution License 4.0, which permits unrestricted use, distribution, and reproduction in any medium, provided the original work is properly cited. 
between different detector components is monitored in situ by light propagation time measurements between light emitters (LED nanobeacons, lasers) and PMTs.

Each DOM is equipped with an internal acoustic piezo detector for the acoustic positioning system [5]. Each line base is equipped with an external hydrophone which has better sensitivity compared to the piezo detectors to reference the line position on the sea bed. The acoustic signal transit time is measured periodically and for three or more emitters position of the receiver can be measured with trilateration. The acoustic emitters are installed in the calibration units [4] that will be placed at known positions on the sea bed. In addition, each calibration unit contains a laser beacon for interline time calibration and water optical properties monitoring, a long-baseline acoustic beacon for DOMs positioning, an hydrophone for their own positioning, and environmental monitoring instruments (conductivity, salinity, temperature, sound velocity, sea currents). The DOM orientation is measured thanks to the internal attitude and heading reference system board.

The key detector components are tested and calibrated at different production steps: PMTs are tested and calibrated in the DarkBox setup. Integrated DOMs are qualified with the GreenBox setups, integrated lines are calibrated with the dark room setups.

\section{PMT characterization}

For the whole infrastructure more than hundred thousand PMTs need to be installed. The Dark Box setup [2] is designed to provide a facility for a fast automatic pre-calibration of 62 PMTs simultaneously which corresponds to the amount of PMTs needed for 2 DOMs. The setup contains a picosecond laser emitter synchronized with the data acquisition system. The laser signal is optically split to reach each PMT with similar power and equal time delay. In KM3NeT a PMT signal is discriminated using a fixed threshold (about $1 / 3$ of the photoelectron signal), and discriminated signal is digitized by time stamping the arrival time with respect to the detector master clock and measuring its duration (Time over Threshold, ToT). The measurement procedure consists of three main phases: HV tuning, darkening and PMT tests with laser.

The tuning is done with $25 \mathrm{~V}$ steps of $\mathrm{HV}$ and ToT is calculated by means of a Gaussian fit function to ToT distribution during $1 \mathrm{~min}$ of data taking for each step. The proper HV, corresponding to ToT $26.4 \mathrm{~ns}$, is found from a parabolic fit to ToT vs HV plot. This ToT value corresponds to the average ToT value estimated on a subset of PMTs with properly calibrated gain $\left(3 \cdot 10^{6}\right)$.

The optimized HV is set to each PMT and the dark rates are monitored during 9 hours. The mean dark count rate is computed by an average over the last $100 \mathrm{~s}$.

The measurements with the laser provide PMT time offsets (mainly due to PMT transit times) and provide an estimation of spurious pulses such as delayed pulses, prepulses and after pulses.

\section{DOM qualification}

After integration DOMs are qualified using the GreenBox setup (Fig. 1).

Since the PMT dark rates can vary after integration due to the contact with structure, glass and gel, their dark rates are rechecked. Also the calibration of the PMTs is checked with a ToT measurement using the dark noise signals.

Radioactive potassium decay in the glass and the gel can be simultaneously seen by the neighbour PMTs in the DOM. This feature is used to verify the PMT mapping and to perform inter PMT time calibration. The example of differences between hit times on two PMTs is shown in Fig. 1. The gaussian peak due to ${ }^{40} \mathrm{~K}$ is centered at 0 for properly intercalibrated PMTs. The area of the peak is proportional the rate of the decays seen simultaneously by two PMTs and it is dependent on the angular distance between them. 

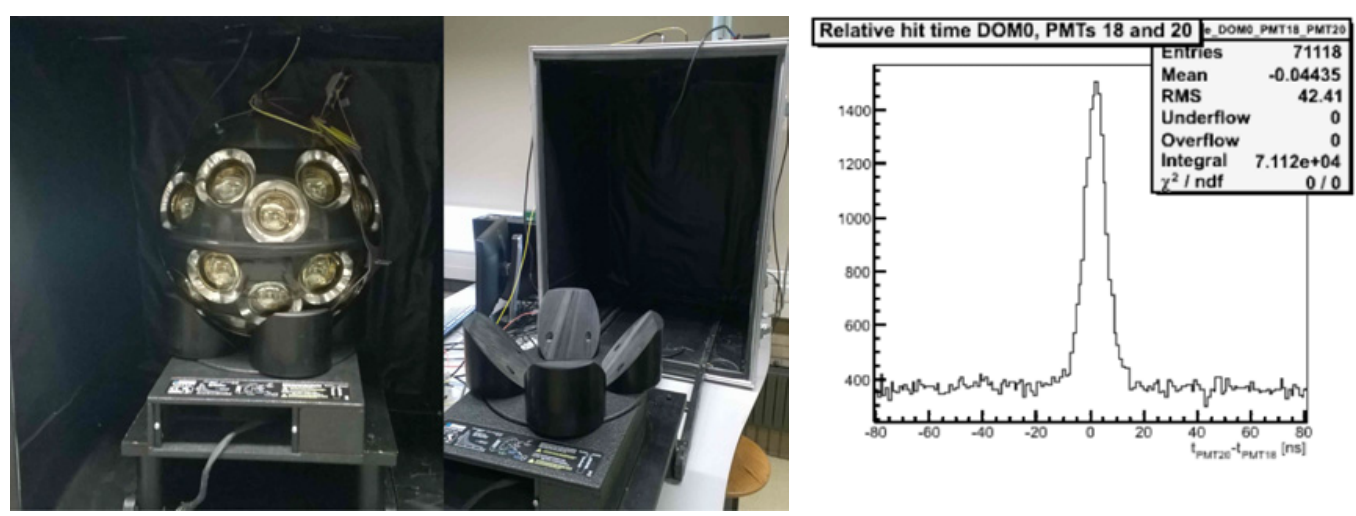

Figure 1. Left: GreenBox images. Right: Distribution of differences between hit times on two PMTs.

The GreenBox is equipped with a laser source which is split to four reference PMTs for the time offsets measurement. This measurement is used later for the time calibration of the line using the same reference PMTs. For the acoustic piezo qualification, the acoustic emitter is placed in the GreenBox. The piezo sensitivity to the signal that will be used for the acoustic positioning is checked.

To calibrate the attitude and heading reference system sensors, inside their working environment, the DOM is placed on a rotating table inside the GreenBox. Three-axis magnetometers can be calibrated by rotating the DOM in orthogonal planes to find the offsets due to magnetic components inside the DOM. The Earth magnetic field designs a sphere in the magnetometers coordinate system during the rotation of the DOM. The sphere is shifted in the presence of the internal magnetic field rotating with the DOM. This offset is found by fitting the rotation measurements with a sphere assumption.

Environmental conditions inside the DOM are checked with an analog pressure manometer. Temperature and humidity are monitored with digital instruments integrated to the slow control system. The integrated LED emitter (nanobeacon) is checked by PMT rate monitoring. DOMs passing the qualification are sent to the line integration sites.

\section{Line qualification}

All the integrated line components are qualified in a dark room setup. Dark room uses laser sources split into 18 equal length channel fibers arriving to the reference PMTs with the same position inside the DOM, one per each DOM. The delay of the laser signal is measured for each DOM to perform the line time calibration (Fig. 2). The delay of about $200 \mathrm{~ns}$ between DOMs corresponds to about $40 \mathrm{~m}$ of the optical fiber connecting the DOMs. The total delay for the light detection by reference PMTs of each DOM is calculated using known laser system propagation time and the cable length connecting the line to the test shore station. After the deployment, this delay will be increased by the known delay introduced by the underwater infrastructure.

The base is calibrated in the laboratory with two short $5 \mathrm{~m}$ cables connecting it to the shore station (time spread due to the different fiber lengths is less than $20 \mathrm{ps}$ ). Time delays in the electronics at the base and at the shore station are calculated and inserted in the White Rabbit calibration tables. These delays ensure that the system estimates properly the cable length and that the reference signals (pulse per second) of the CLB and the shore station are synchronised (Fig. 2). The same calibration is repeated with reference CLB which has a frozen firmware to monitor possible delay changes due to CLB firmware upgrades in the base or in the shore station. 

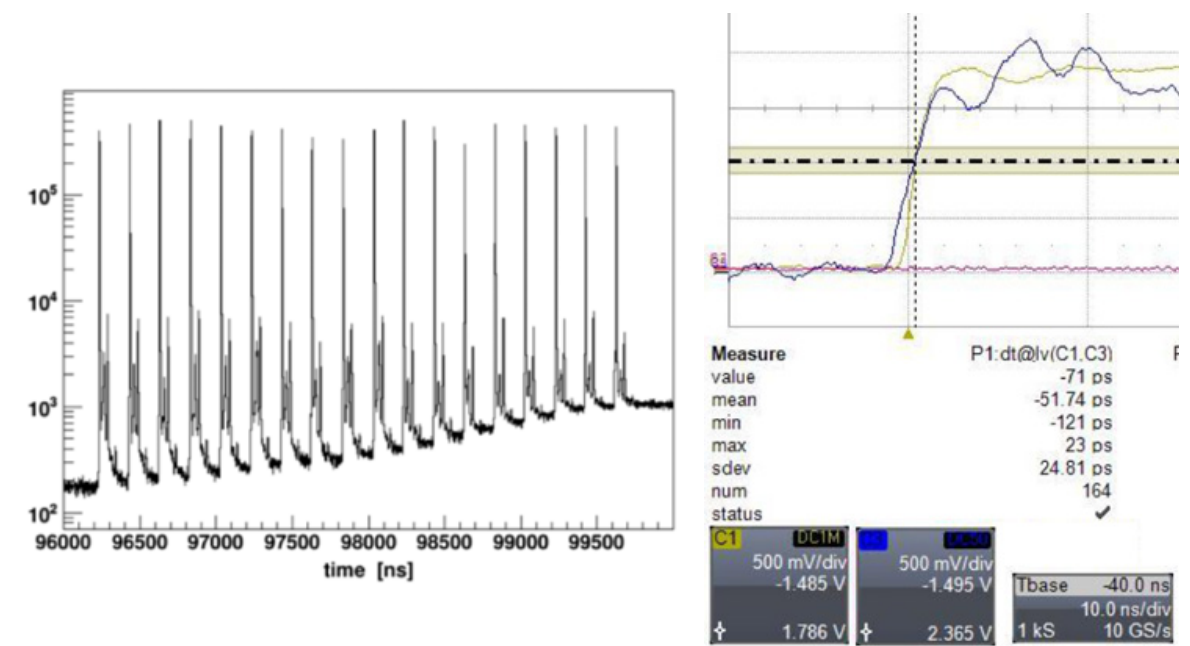

Figure 2. Left: laser photons detection time for the reference PMTs. Each peak corresponds to one DOM. Right: pulse per second signal for the shore station (yellow) and the line base (blue) synchronised at sub-ns level.

The dark room is equipped with several synchronized acoustic emitters which can be placed nearby the DOMs. The delay of the acoustic signal arrival is measured for DOM's piezos. For this purpose a correlation analysis is performed in which the emitted signal with a known shape is compared to the detected signal plus noise.

\section{Calibration after deployment}

Several instruments will be used to perform the verification and adjustment of the time calibration insitu. ${ }^{40} \mathrm{~K}$ decay in water will be used for PMT time delay calibration between PMTs in the same DOM. The nanobeacons installed in each DOM provide light detection from one DOM to the neighbor DOMs for their inter calibration. Vertical atmospheric muons provide alternative inter-DOM calibration. Laser beacons installed on the Calibration Units provide light detection by DOMs of different lines for the time calibration between the lines. Positioning of the DOMs will be performed thanks to the acoustic instruments installed in the DOMs, DU bases and calibration units.

\section{Summary}

The KM3NeT detector is under construction. Detector components are qualified and calibrated on different production stages: PMT delivery, DOM integration, line integration, deployment and in-situ operation. These procedures were tested and optimised during first DU line integration.

\section{References}

[1] R. Coniglione in this conference, D. Samtleben in this conference

[2] C. M. Mollo, this conference

[3] G. Kieft, this conference

[4] A. Kouchner, B. Baret, this conference

[5] F. Simeone, this conference 